\title{
UNIQUENESS OF RENORMALIZED SOLUTIONS TO NONLINEAR PARABOLIC PROBLEMS WITH LOWER ORDER TERMS
}

\author{
ROSARIA DI NARDO, FILOMENA FEO, AND OLIVIER GUIBÉ
}

\begin{abstract}
In this paper we prove uniqueness results for renormalized solutions to a class of nonlinear parabolic problems.
\end{abstract}

\section{INTRODUCTION}

In the present paper we investigate the uniqueness of the following class of nonlinear parabolic problems

$$
\left\{\begin{array}{rr}
\frac{\partial u}{\partial t}-\operatorname{div}(a(x, t, u, \nabla u)) & \text { in } Q_{T} \\
& +\operatorname{div}(K(x, t, u))+H(x, t, \nabla u)=f-\operatorname{div} g \\
u(x, t)=0 & \text { on } \partial \Omega \times(0, T) \\
u(x, 0)=u_{0}(x) & \text { in } \Omega,
\end{array}\right.
$$

where $Q_{T}$ is the cylinder $\Omega \times(0, T), \Omega$ is a bounded open subset of $\mathbb{R}^{N}$, $T>0, p>1$ and $N \geq 2$. Moreover $-\operatorname{div}(a(x, t, u, \nabla u))$ is a Leray-Lions operator which is coercive and grows like $|\nabla u|^{p-1}$ with respect to $\nabla u$. The function $K$ and $H$ are Carathéodory functions with suitable assumptions (see Theorems 3.1, 3.2 and 3.3). Finally $f \in L^{1}\left(Q_{T}\right), g \in\left(L^{p^{\prime}}\left(Q_{T}\right)\right)^{N}$ and $u_{0} \in L^{1}(\Omega)$.

The difficulties connected to existence and uniqueness of the solution to this problem are due to the $L^{1}$ data and to the presence of the two terms $K$ and $H$ which can induce a lack of coercivity.

For $L^{1}$ data and $p>2-\frac{1}{N+1}$ the existence of a weak solution to Problem (1.1) (which belongs to $L^{m}\left((0, T) ; W_{0}^{1, m}(\Omega)\right)$ with $m<\frac{p(N+1)-N}{N+1}$ ) was proved in [7] (see also [6]) when $K \equiv H \equiv 0$ and in [22] when $K \equiv 0$. It is well known that this weak solution is not unique in general (see [25] for a counter-example in the stationary case). In the present paper we use the framework of renormalized solutions which provides uniqueness and stability properties.

The notion of renormalized solution was introduced in [14, 15] for first order equations and has been adapted for elliptic problems with $L^{1}$ data

2000 Mathematics Subject Classification. 35K55, 35K20, 35R05 .

Key words and phrases. Uniqueness result, Nonlinear parabolic equations, renormalized solution, integrable data. 
in [18, 19]) and with bounded measure data in [10]. This notion was also developed for parabolic equation with $L^{1}$ data in [3, 4] (see also [21] for measure data). Recall that the equivalent notion of entropy solution for $L^{1}$ data was also developed for elliptic equation in [1] (see also [23] in the parabolic case).

In the case where $H \equiv 0$ and where the function $K(x, t, u)$ is independent on the $(x, t)$ variable and continuous, the existence of a renormalized solution to Problem (1.1) is proved in [4]. The case $H \equiv 0, g \equiv 0$ (and where $K$ depends on $(x, t)$ and $u$ ) is investigated in [11]. In [12] the authors prove the existence of a renormalized solution for the complete operator.

As far as the uniqueness of renormalized solution to parabolic equation is concerned, we refer mainly to [3, 4, 9] where in short the function $K$ does not depend on the $(x, t)$ variable and where $H \equiv 0$ (see also [5] for Stefan problem with $L^{1}$ data). In particular, when $H \equiv 0$ and under a local Lipschitz assumption on $a(x, t, r, \xi)$ and on $K(r)$ with respect to $r$ the authors prove in [4] that the renormalized solution to Problem (1.1) is unique. With respect to the mentioned references, the main novelty of the present paper is to present uniqueness results to parabolic equations (1.1) with the two terms $-\operatorname{div}(K(x, t, u))$ and $H(x, t, \nabla u)$. The first result (see Theorem 3.1) deals with the case $H \equiv 0$ and establishes the uniqueness of the renormalized solution to Problem (1.1) under a local Lipschitz condition on $a(x, t, r, \xi)$ and $K(x, t, r)$ with respect to $r$. The proof uses the techniques developed in [4] and the dependence of the function $K$ with respect to the $(x, t)$ variable leads to additional difficulties here. Such difficulties are overcome by a technical lemma (see Lemma 4.1) which specifies the asymptotic behavior of some terms which appear in the uniqueness process. The second result (see Theorem 3.2 for $p \geq 2$ and Theorem 3.3 for $2-\frac{1}{N+1}<p<2$ ) addresses Equation (1.1) with the presence of the two terms $-\operatorname{div}(K(x, t, u))$ and $H(x, t, \nabla u)$. Under more restrictive assumptions on $a$ and under global Lipschitz type condition on $K(x, t, s)$ with respect to $s$ and $H(x, t, \xi)$ with respect to $\xi$ we show the uniqueness of the renormalized solution. The proof uses two technical lemmas (Lemma 4.1 and Lemma 4.2) and the techniques developed in 12 for the existence of a solution to Problem (1.1) (see also [22]). We underline that we don't make any assumptions on the smallness of the coefficients. Indeed for the analogous elliptic equation with two lower order terms (see e.g. [16] and [13]) it is necessary to assume that one of the terms $K$ or $H$ is small enough in order to obtain an existence and uniqueness result.

The paper is organized as follows. In Section 2 we present the assumptions on the data and we recall the definition of a renormalized solution to Problem (1.1). In Section 3 we state the main results of the present paper. Section 4 is devoted to the proof of the uniqueness results. 


\section{Assumptions and Definitions}

In this section we recall the definition of a renormalized solution to nonlinear parabolic problems with lower order terms and $L^{1}(\Omega \times(0, T))+$ $L^{p^{\prime}}\left((0, T) ; W^{-1, p^{\prime}}(\Omega)\right)$ data.

More precisely we consider the following problem

$$
\left\{\begin{array}{rr}
\frac{\partial u}{\partial t}-\operatorname{div}(a(x, t, u, \nabla u)) & \text { in } Q_{T} \\
& +\operatorname{div}(K(x, t, u))+H(x, t, \nabla u)=f-\operatorname{div} g \\
u(x, t)=0 & \text { on } \partial \Omega \times(0, T) \\
u(x, 0)=u_{0}(x) & \text { in } \Omega,
\end{array}\right.
$$

where $Q_{T}$ is the cylinder $\Omega \times(0, T), \Omega$ is a bounded open subset of $\mathbb{R}^{N}$ with boundary $\partial \Omega, T>0, p>1$ and $N \geq 2$.

The following assumptions hold true:

- $a: Q_{T} \times \mathbb{R} \times \mathbb{R}^{N} \rightarrow \mathbb{R}^{N}$ is Carathéodory function such that

and

$$
a(x, t, s, \xi) \xi \geq \alpha_{0}|\xi|^{p}, \quad \alpha_{0}>0,
$$

$$
(a(x, t, s, \xi)-a(x, t, s, \bar{\xi}), \xi-\bar{\xi})>0,
$$

for a.e. $(x, t) \in Q_{T}$, for any $s \in \mathbb{R}$ and any $\xi, \bar{\xi} \in \mathbb{R}^{N}$ with $\xi \neq \bar{\xi}$.

Moreover for any $k>0$ there exists $\beta_{k}>0$ and $h_{k} \in L^{p^{\prime}}\left(Q_{T}\right)$ such that

$$
|a(x, t, s, \xi)| \leq h_{k}+\beta_{k}|\xi|^{p-1}, \quad \text { for every } s \text { such that }|s| \leq k,
$$

for a.e. $(x, t) \in Q_{T}$ and any $\xi \in \mathbb{R}^{N}$;

- $K: Q_{T} \times \mathbb{R} \rightarrow \mathbb{R}^{N}$ is a Carathéodory function such that

$$
|K(x, t, s)| \leq c(x, t)\left(|s|^{\gamma}+1\right),
$$

with

$$
\gamma=\frac{N+2}{N+p}(p-1) \text { and } c \in L^{r}\left(Q_{T}\right) \text { with } r \geq \frac{N+p}{p-1},
$$

for a.e. $(x, t) \in Q_{T}$, for every $s \in \mathbb{R}$;

- $H: Q_{T} \times \mathbb{R}^{N} \rightarrow \mathbb{R}$ is a Carathéodory function such that

with

$$
|H(x, t, \xi)| \leq b(x, t)\left(|\xi|^{\delta}+1\right),
$$

$$
\delta=\frac{N(p-1)+p}{N+2} \text { and } b \in L^{N+2,1}\left(Q_{T}\right),
$$

for a.e. $(x, t) \in Q_{T}$ and for every $\xi \in \mathbb{R}^{N}$.

Moreover we assume that

$$
\begin{gathered}
f \in L^{1}\left(Q_{T}\right), \\
g \in\left(L^{p^{\prime}}\left(Q_{T}\right)\right)^{N}
\end{gathered}
$$


and

$$
u_{0} \in L^{1}(\Omega) .
$$

Under these assumptions, the above problem does not admit, in general, a solution in the sense of distribution since we cannot expect to have the fields $a(x, t, u, \nabla u), K(x, t, u)$ in $\left(L_{l o c}^{1}\left(Q_{T}\right)\right)^{N}$ and $H(x, t, \nabla u)$ in $L_{l o c}^{1}\left(Q_{T}\right)$. For this reason in the present paper we consider the framework of renormalized solutions.

For any $k>0$ we denote by $T_{k}$ the truncation function at height $\pm k$, $T_{k}(s)=\max (-k, \min (k, s))$ for any $s \in \mathbb{R}$.

We recall the definition of a renormalized solution (see [3, 4]) to Problem (2.1).

Definition 2.1. A real function $u$ defined in $Q_{T}$ is a renormalized solution of (2.1) if it satisfies the following conditions:

$$
\begin{gathered}
u \in L^{\infty}\left((0, T) ; L^{1}(\Omega)\right), \\
T_{k}(u) \in L^{p}\left((0, T) ; W_{0}^{1, p}(\Omega)\right), \text { for any } k>0, \\
\lim _{n \rightarrow+\infty} \frac{1}{n} \int_{\left\{(x, t) \in Q_{T}:|u(x, t)| \leq n\right\}} a(x, t, u, \nabla u) \nabla u d x d t=0,
\end{gathered}
$$

and if for every function $S \in W^{2, \infty}(\mathbb{R})$ which is piecewise $C^{1}$ and such that $S^{\prime}$ has a compact support

$$
\begin{aligned}
\frac{\partial S(u)}{\partial t}- & \operatorname{div}\left(a(x, t, u, \nabla u) S^{\prime}(u)\right)+S^{\prime \prime}(u) a(x, t, u, \nabla u) \nabla u \\
& +\operatorname{div}\left(K(x, t, u) S^{\prime}(u)\right)-S^{\prime \prime}(u) K(x, t, u) \nabla u \\
& +H(x, t, \nabla u) S^{\prime}(u)=f S^{\prime}(u)-(\operatorname{div} g) S^{\prime}(u) \text { in } \mathcal{D}^{\prime}\left(Q_{T}\right)
\end{aligned}
$$

and

$$
S(u)(t=0)=S\left(u_{0}\right) \quad \text { in } \Omega .
$$

Remark 1. It is well known that conditions (2.12) and (2.13) allow to define $\nabla u$ almost everywhere in $Q_{T}$ : for any $k>0$ we have $\nabla T_{k}(u)=$ $\chi_{\{|u|<k\}} \nabla u$ a.e in $Q_{T}$ where $\chi_{\{|u|<k\}}$ denotes the characteristic function of the set $\{(x, t):|u(x, t)|<k\}$. We notice that equation (2.15) can be formally obtained through pointwise multiplication of (2.1) by $S^{\prime}(u)$ and all terms except $S(u)_{t}$ in (2.15) belong to $L^{1}\left(Q_{T}\right)+L^{p^{\prime}}\left((0, T) ; W^{-1, p^{\prime}}(\Omega)\right)$ since $T_{k}(u) \in L^{p}\left((0, T) ; W_{0}^{1, p}(\Omega)\right)$, for any $k>0$ and $S^{\prime}$ has a compact support. It follows that (2.15) has a meaning in $\mathcal{D}^{\prime}\left(Q_{T}\right)$ and that the initial condition (2.16) makes sense. At last condition (2.14) gives additional information on $\nabla u$ for large value of $|u|$. 
We use in the present paper the two Lorentz spaces $L^{q, 1}\left(Q_{T}\right)$ and $L^{q, \infty}\left(Q_{T}\right)$, see for example [17, 20] for references about Lorentz spaces $L^{q, s}$. If $f^{*}$ denotes the decreasing rearrangement of a measurable function $f$,

$$
f^{*}(r)=\inf \left\{s \geq 0: \text { meas }\left\{(x, t) \in Q_{T}:|f(x, t)|>s\right\}<r\right\},
$$

with $r \in\left[0, \operatorname{meas}\left(Q_{T}\right)\right], L^{q, 1}\left(Q_{T}\right)$ is the space of Lebesgue measurable functions such that

$$
\|f\|_{L^{q, 1}\left(Q_{T}\right)}=\left(\int_{0}^{\operatorname{meas}\left(Q_{T}\right)} f^{*}(r) r^{\frac{1}{q}} \frac{d r}{r}\right)<+\infty
$$

while $L^{q, \infty}\left(Q_{T}\right)$ is the space of Lebesgue measurable functions such that

$$
\|f\|_{L^{q, \infty}\left(Q_{T}\right)}=\sup _{r>0} r\left[\operatorname{meas}\left\{(x, t) \in Q_{T}:|f(x, t)|>r\right\}\right]^{1 / q}<+\infty .
$$

If $1<q<+\infty$ we have the generalized Hölder inequality

$$
\left\{\begin{array}{l}
\forall f \in L^{q, \infty}\left(Q_{T}\right), \forall g \in L^{q^{\prime}, 1}\left(Q_{T}\right), \\
\int_{Q_{T}}|f g| \leq\|f\|_{L^{q, \infty}\left(Q_{T}\right)}\|g\|_{L^{q^{\prime}, 1}\left(Q_{T}\right)}
\end{array}\right.
$$

Under the assumptions (2.2)-(2.11) the existence of a renormalized solution to Problem (2.1) is established in [12] and it is well known that (2.12)(2.14) lead to

$$
|\nabla u| \in L^{\frac{N(p-1)+p}{N+1}, \infty}\left(Q_{T}\right)
$$

and

$$
u \in L^{\frac{N(p-1)+p}{N}, \infty}\left(Q_{T}\right) .
$$

Moreover the growth assumptions (2.5), (2.7) on $K$ and $H$, the regularities (2.6), (2.8) of $c$ and $b$ together with (2.12) and (2.14) allow to prove (see [12]) that any renormalized solution to Problem (2.1) verifies

$$
H(x, t, \nabla u) \in L^{1}\left(Q_{T}\right)
$$

and

$$
\lim _{n \rightarrow+\infty} \frac{1}{n} \int_{\left\{(x, t) \in Q_{T} ;|u(x, t)|<n\right\}}|K(x, t, u)||\nabla u| d x d t=0 .
$$

Properties (2.20) and (2.21) are crucial to obtain uniqueness results.

Notation. Throughout the paper, for the sake of shortness if $u$ is a measurable function defined on $Q_{T}$, we denote by $\{|u| \leq k\}$ (resp. $\quad\{|u|<$ $k\}$ ) the measurable subset $\left\{(x, t) \in Q_{T} ;|u(x, t)| \leq k\right\}$ (resp. $\{(x, t) \in$ $\left.Q_{T} ;|u(x, t)|<k\right\}$. Moreover the explicit dependence in $x$ and $t$ of the functions $a, K$ and $H$ will be omitted so that $a(x, t, u, \nabla u)=a(u, \nabla u)$, $K(u)=K(x, t, u)$ and $H(\nabla u)=H(x, t, \nabla u)$. 


\section{Statement of the Results}

3.1. First case: $H \equiv 0$. In order to prove uniqueness result in the case $H(x, t, \xi)=0$ we assume the further condition that $a(x, t, s, \xi)$ and $K(x, t, s)$ are locally continuous Lipschitz with respect to $s$ : for any compact set $C$ of $\mathbb{R}$, there exists $L_{C}$ belonging to $L^{p^{\prime}}\left(Q_{T)}\right)$ and $\gamma_{C}>0$ such that $\forall s, \bar{s} \in C$

$$
\begin{gathered}
|a(x, t, s, \xi)-a(x, t, \bar{s}, \xi)| \leq\left(L_{C}(x, t)+\gamma_{C}|\xi|^{p-1}\right)|s-\bar{s}| \\
|K(x, t, s)-K(x, t, \bar{s})| \leq L_{C}(x, t)|s-\bar{s}|
\end{gathered}
$$

for almost every $(x, t) \in Q_{T}$ and for every $\xi \in \mathbb{R}^{N}$.

The main result of this subsection is the following theorem.

Theorem 3.1. Under the assumptions (2.2)-(2.6), (2.9)-(2.11), (3.1) and (3.2), the renormalized solution to Problem (2.1) is unique.

3.2. Second case: general operator. In order to prove uniqueness result for Problem (2.1) with the term $H(x, t, \nabla u)$ we assume in this subsection that the function $a$ is independent of $r$ and is strongly monotone (see assumptions (3.5) in Theorem 3.2 and (3.7) in Theorem 3.3).

Moreover the functions $K(x, t, s)$ (resp. $H(x, t, \xi)$ ) is locally Lipschitz continuous with respect to $s$ (resp. $\xi$ ) with a global control of the Lipschitz coefficient:

$$
|K(x, t, s)-K(x, t, \bar{s})| \leq c(x, t)(1+|s|+|\bar{s}|)^{\tau}|s-\bar{s}| \quad \tau \geq 0
$$

and

$$
|H(x, t, \xi)-H(x, t, \bar{\xi})| \leq b(x, t)(1+|\xi|+|\bar{\xi}|)^{\sigma}|\xi-\bar{\xi}| \quad \sigma \geq 0
$$

for a.e. $(x, t) \in Q_{T}$, for every $s, \bar{s} \in \mathbb{R}$, for every $\xi, \bar{\xi} \in \mathbb{R}^{N}$ with $c \in L^{r, 1}\left(Q_{T}\right)$ and $b \in L^{\lambda, 1}\left(Q_{T}\right)$ where $r$ and $\lambda$ belong to suitable intervals (see Theorems 3.3 and 3.4 )

We investigate the case $p \geq 2$ and the case $2-\frac{1}{N+1}<p<2$ in two different results.

Theorem 3.2. Let $p \geq 2$. Let us assume that (2.2)-(2.11) hold and that the function $a$ is independent of $r$ and satisfies

$$
(a(x, t, \xi)-a(x, t, \bar{\xi})) \cdot(\xi-\bar{\xi}) \geq \beta(1+|\xi|+|\bar{\xi}|)^{p-2}|\xi-\bar{\xi}|^{2}
$$

for a.e. $(x, t) \in Q_{T}$, for every $\xi, \bar{\xi} \in \mathbb{R}^{N}$ with $\xi \neq \bar{\xi}$ and $\beta>0$.

Moreover we assume that (3.3) and (3.4) are satisfied with

$$
\begin{cases}r \geq N+2, & 0 \leq \tau \leq \frac{N(p-1)+p}{N}\left(\frac{1}{N+2}-\frac{1}{r}\right), \\ \lambda \geq N+2, & 0 \leq \sigma \leq \frac{N(p-1)+p}{N+1}\left(\frac{1}{N+2}-\frac{1}{\lambda}\right) .\end{cases}
$$

Then the renormalized solution to Problem (2.1) is unique. 
Theorem 3.3. Let $2-\frac{1}{N+1}<p<2$. Let us assume that (2.2) $-(2.4)$, (2.9) (2.11) hold and that the function $a$ is independent of $r$ and satisfies

$$
(a(x, t, \xi)-a(x, t, \bar{\xi})) \cdot(\xi-\bar{\xi}) \geq \beta \frac{|\xi-\bar{\xi}|^{2}}{(|\xi|+|\bar{\xi}|)^{2-p}}
$$

for a.e. $(x, t) \in Q_{T}$, for every $\xi, \bar{\xi} \in \mathbb{R}^{N}$ with $\xi \neq \bar{\xi}$ and $\beta>0$. Moreover we assume that (3.3) and (3.4) are satisfied with

$$
\left\{\begin{array}{l}
r>\frac{p(N+1)-N}{(p-1)(N+1)-N}, \\
0 \leq \tau<\frac{N(p-1)+p}{N}\left(\frac{(p-1)(N+1)-N}{p(N+1)-N}-\frac{1}{r}\right), \\
\lambda>\frac{p(N+1)-N}{(p-1)(N+1)-N}, \\
0 \leq \sigma<\frac{N(p-1)+p}{N+1}\left(\frac{(p-1)(N+1)-N}{p(N+1)-N}-\frac{1}{\lambda}\right) .
\end{array}\right.
$$

Then the renormalized solution to Problem (2.1) is unique.

Remark 2. Let us compare the assumptions (2.5) and (2.7) on the growth condition and the assumptions (3.3) and (3.4) on the locally Lipschitz continuity made on $K(x, t, s)$ and $H(x, t, \xi)$ respectively. Observe that assumption (3.3) (3.4) respectively) implies a growth condition on $K(x, t, s)$ (on $H(x, t, \xi)$ respectively), that can be more restrictive than (2.5) (2.7) respectively), depending on the value of $\tau$ ( $\sigma$ respectively).

The model function $a(x, t, \xi)$ which satisfies assumptions (2.4), (3.5) or (3.7) is

$$
a(x, t, \xi)=\left\{\begin{array}{lll}
a(x, t)|\xi|^{p-2} \xi & \text { if } \quad 2-\frac{1}{N+1}<p<2 \\
a(x, t)\left(1+|\xi|^{2}\right)^{\frac{p-2}{2}} \xi & \text { if } \quad p \geq 2
\end{array}\right.
$$

where $a(x, t) \in L^{\infty}\left(Q_{T}\right)$ and $a(x, t)>\beta>0$.

Examples of functions $K(x, t, s)$ and $H(x, t, \xi)$ are given by

$$
K(x, t, s)=c(x, t)(1+|s|)^{\bar{\gamma}} \quad \text { with } \quad \bar{\gamma}=\min \{\gamma, \tau+1\}
$$

and

$$
H(x, t, \xi)=b(x, t)(1+|\xi|)^{\bar{\delta}} \quad \text { with } \quad \bar{\delta}=\min \{\delta, \sigma+1\}
$$

where $c(x, t) \in L^{r, 1}\left(Q_{T}\right)$ and $b(x, t) \in L^{\lambda, 1}\left(Q_{T}\right)$ with

$$
\left\{\begin{array}{lll}
r>\frac{p(N+1)-N}{(p-1)(N+1)-N} & \text { if } & 2-\frac{1}{N+1}<p<2, \\
r \geq N+2 & \text { if } & p \geq 2
\end{array}\right.
$$

and

$$
\left\{\begin{array}{lll}
\lambda>\frac{p(N+1)-N}{(p-1)(N+1)-N} & \text { if } & 2-\frac{1}{N+1}<p<2 \\
\lambda \geq N+2 & \text { if } & p \geq 2
\end{array}\right.
$$




\section{Proof of the Results}

This section is devoted to prove Theorems 3.1, 3.2 and 3.3. We start by a technical lemma which is similar to Lemma 6 of [4 for a different parabolic equation with $L^{1}$ data. It allows to control the behavior of some quantities which appear in the uniqueness process. We stress that our proof is different to the one in [4] and uses only the fact that two renormalized solutions of (2.1) verify (2.14) and (2.21) (notice that (2.21) is a consequence of (2.14) and the growth assumption of $K)$. See also [2 for such a generalization on parabolic equation of the kind $\frac{\partial b(u)}{\partial t}-\operatorname{div}(a(x, t, u, \nabla u))=f+\operatorname{div} g$.

Lemma 4.1. Under the assumptions (2.2)-(2.11), let $u$ and $v$ be two renormalized solutions to Problem (2.1). Let us define for any $0<k<s$

$$
\begin{aligned}
& \Gamma(u, v, s, k)= \\
& \quad \int_{\{s-k<|u|<s+k\}}\left(a(u, \nabla u) \nabla u+|K(u)||\nabla u|+|g|^{p^{\prime}}\right) d x d t \\
& +\int_{\{s-k<|v|<s+k\}}\left(a(v, \nabla v) \nabla v+|K(v)||\nabla v|+|g|^{p^{\prime}}\right) d x d t
\end{aligned}
$$

and for any $0<r<s$

$$
\begin{aligned}
\Theta(u, v, s, r)= & \int_{\{s-r<|u|<s\}}(a(u, \nabla u) \nabla u+|K(u)||\nabla u|) d x d t \\
& +\int_{\{s-r<|v|<s\}}(a(v, \nabla v) \nabla v+|K(v)||\nabla v|) d x d t .
\end{aligned}
$$

Then we have for any $r>0$

$$
\liminf _{s \rightarrow \infty}\left(\limsup _{k \rightarrow 0} \frac{1}{k} \Gamma(u, v, s, k)+\Theta(u, v, s, r)\right)=0 .
$$

Proof. We argue by contradiction. Let $r$ be a positive real number. If the thesis of lemma is not true, let $\varepsilon_{0}>0$ and let $n_{0}$ be an integer such that for every real number $s \geq n_{0}$ we have

$$
\limsup _{k \rightarrow 0} \frac{1}{k} \Gamma(u, v, s, k)+\Theta(u, v, s, r) \geq \varepsilon_{0} .
$$

Let us consider the function

$$
\begin{aligned}
F(s)= & \int_{\{|u|<s\}}\left(a(u, \nabla u) \nabla u+|K(u)||\nabla u|+|g|^{p^{\prime}}\right) d x d t \\
& +\int_{\{|v|<s\}}\left(a(v, \nabla v) \nabla v+|K(v)||\nabla v|+|g|^{p^{\prime}}\right) d x d t .
\end{aligned}
$$

Due to (2.2) the function $F$ is monotone increasing. It follows (see e.g. [24]) that $F$ is derivable almost everywhere, with $F^{\prime}$ measurable, and that we have for any $s>\eta>0$

$$
F(s)-F(\eta) \geq \int_{\eta}^{s} F^{\prime}(\xi) d \xi
$$


and for almost any $s>0$

$$
\begin{aligned}
& F^{\prime}(s)= \\
& \quad \frac{1}{2} \limsup _{k \rightarrow 0} \frac{1}{k}\left[\int_{\{s-k \leq|u|<s+k\}}\left(a(u, \nabla u) \nabla u+|K(u)||\nabla u|+|g|^{p^{\prime}}\right) d x d t\right. \\
& \left.\quad+\int_{\{s-k \leq|v|<s+k\}}\left(a(v, \nabla v) \nabla v+|K(v)||\nabla v|+|g|^{p^{\prime}}\right) d x d t\right] .
\end{aligned}
$$

Moreover due to (2.14) and (2.21) and since $g$ belongs to $\left(L^{p^{\prime}}\left(Q_{T}\right)\right)^{N}$ we have

$$
\lim _{s \rightarrow+\infty} \frac{F(s)}{s}=0
$$

Due to the definition of $\Gamma(u, v, s, k)$, inequality (4.4) leads to

$$
F^{\prime}(\xi)+\frac{1}{2} \Theta(u, v, \xi, r) \geq \frac{\varepsilon_{0}}{2}
$$

for almost $\xi \geq n_{0}$. From (4.5) it follows that

$$
\frac{1}{s-n_{0}}\left(F(s)+\frac{1}{2} \int_{n_{0}}^{s} \Theta(u, v, \xi, r) d \xi\right) \geq \frac{\varepsilon_{0}}{2}+\frac{F\left(n_{0}\right)}{s-n_{0}} \quad \text { for } s>n_{0} .
$$

Due to the definition of $\Theta$, a few computations give

$$
\begin{aligned}
\int_{n_{0}}^{s} \Theta(u, v, \xi, r) d \xi \leq r & \left(\int_{\{|u|<s\}}|K(u)||\nabla u| d x d t\right. \\
& \left.+\int_{\{|v|<s\}}|K(v)||\nabla v| d x d t\right) .
\end{aligned}
$$

It follows that

$$
\begin{aligned}
& \frac{1}{s-n_{0}}\left(F(s)+\frac{r}{2}\left(\int_{\{|u|<s\}}|K(u)||\nabla u| d x d t\right.\right. \\
& \left.\left.\quad+\int_{\{|v|<s\}}|K(v)||\nabla v| d x d t\right)\right) \geq \frac{\varepsilon_{0}}{2}+\frac{F\left(n_{0}\right)}{s-n_{0}} \quad \text { for } s>n_{0} .
\end{aligned}
$$

The last inequality contradicts (2.14) and (4.6).

Proof of Theorem [3.1. The strategy is similar to the proof of Theorem 2 in [4]. It consists to define a smooth approximation $T_{s}^{\sigma}$ of the truncation $T_{s}$ and to consider two renormalized solutions $u$ and $v$ to Problem (2.1) for the same data $f, g$ and $u_{0}$. In Step 1 we plug the test function $\frac{1}{k} T_{k}\left(T_{s}^{\sigma}(u)-T_{s}^{\sigma}(v)\right)$ in the difference of the equations (2.15) for $u$ and $v$ in which we have taken $S=T_{s}^{\sigma}$. This process then leads to equation (4.9). In Step 2 we study the behavior of the terms of (4.9) with respect to $\sigma, k$ and $s$, with the help of Lemma 4.1. In Step 3 we then pass to the limit when $\sigma \rightarrow 0, k \rightarrow 0$ and $s \rightarrow+\infty$. 
Step 1. Let $u$ and $v$ be two renormalized solutions to Problem (2.1) for the same data $f, g$ and $u_{0}$. For every real number $s>0$ and $\sigma>0$, let $T_{s}^{\sigma}$ be the function defined by

$$
\begin{cases}T_{s}^{\sigma}(0)=0, & \text { for }|r|<s \\ \left(T_{s}^{\sigma}\right)^{\prime}(r)=1 & \text { for } s \leq|r| \leq s+\sigma \\ \left(T_{s}^{\sigma}\right)^{\prime}(r)=\frac{1}{\sigma}(s+\sigma-|r|) & \text { for }|r|>s+\sigma \\ \left(T_{s}^{\sigma}\right)^{\prime}(r)=0 & \end{cases}
$$

We take $S=T_{s}^{\sigma}$ in (2.15) for $u$ and $v$. Subtracting these two equations and plugging the test function $\frac{1}{k} T_{k}\left(T_{s}^{\sigma}(u)-T_{s}^{\sigma}(v)\right)$, we obtain upon integration on $(0, t)$, that for every $k>0, s>0, \sigma>0$,

$$
\begin{aligned}
\frac{1}{k} \int_{0}^{t}\left\langle\frac { \partial } { \partial t } \left[ T_{s}^{\sigma}(u)-\right.\right. & \left.\left.T_{s}^{\sigma}(v)\right], T_{k}\left(T_{s}^{\sigma}(u)-T_{s}^{\sigma}(v)\right)\right\rangle d \tau \\
& +\frac{1}{k}\left(A_{s, k}^{\sigma}(t)+\widetilde{A}_{s, k}^{\sigma}(t)\right) \\
= & \frac{1}{k}\left(C_{s, k}^{\sigma}(t)+\widetilde{C}_{s, k}^{\sigma}(t)+F_{s, k}^{\sigma}(t)+G_{s, k}^{\sigma}(t)+\widetilde{G}_{s, k}^{\sigma}(t)\right)
\end{aligned}
$$

for almost any $t \in(0, T)$, where $\langle$,$\rangle denotes the duality between L^{1}(\Omega)+$ $W^{-1, p^{\prime}}(\Omega)$ and $L^{\infty}(\Omega) \cap W_{0}^{1, p}(\Omega)$ and where

$$
\begin{aligned}
A_{s, k}^{\sigma}(t)= & \int_{0}^{t} \int_{\Omega}\left[\left(T_{s}^{\sigma}\right)^{\prime}(u) a(u, \nabla u)-\left(T_{s}^{\sigma}\right)^{\prime}(v) a(v, \nabla v)\right] \\
& \times \nabla T_{k}\left(T_{s}^{\sigma}(u)-T_{s}^{\sigma}(v)\right) d x d \tau
\end{aligned}
$$


UNIQUENESS OF RENORMALIZED SOLUTIONS TO NONLINEAR PARABOLIC ... 11

$$
\begin{aligned}
F_{s, k}^{\sigma}(t) & =\int_{0}^{t} \int_{\Omega} f\left[\left(T_{s}^{\sigma}\right)^{\prime}(u)-\left(T_{s}^{\sigma}\right)^{\prime}(v)\right] T_{k}\left(T_{s}^{\sigma}(u)-T_{s}^{\sigma}(v)\right) d x d \tau, \\
G_{s, k}^{\sigma}(t) & =\int_{0}^{t} \int_{\Omega} g\left[\left(T_{s}^{\sigma}\right)^{\prime}(u)-\left(T_{s}^{\sigma}\right)^{\prime}(v)\right] \nabla T_{k}\left(T_{s}^{\sigma}(u)-T_{s}^{\sigma}(v)\right) d x d \tau, \\
\widetilde{G}_{s, k}^{\sigma}(t) & =\int_{0}^{t} \int_{\Omega} g \nabla\left[\left(T_{s}^{\sigma}\right)^{\prime}(u)-\left(T_{s}^{\sigma}\right)^{\prime}(v)\right] T_{k}\left(T_{s}^{\sigma}(u)-T_{s}^{\sigma}(v)\right) d x d \tau .
\end{aligned}
$$

In order to pass to the limit in (4.9) when $\sigma \rightarrow 0, k \rightarrow 0$ and $s \rightarrow+\infty$, we observe that by (4.8) we have for almost any $t \in(0, T)$

$$
T_{s}^{\sigma}(u) \rightarrow T_{s}(u) \text { in } L^{p}\left((0, t) ; W_{0}^{1, p}(\Omega)\right) \text { and a.e. in } \Omega \times(0, t)
$$

and

$$
\left(T_{s}^{\sigma}\right)^{\prime}(u) \rightarrow \chi_{\{|u| \leq s\}} \text { in } L^{q}(\Omega \times(0, t)) \text { and a.e. in } \Omega \times(0, t)
$$

for every $1<q<+\infty$ for fixed $s>0$ when $\sigma$ tends to zero.

By defining $\Psi_{k}(r)=\int_{0}^{r} T_{k}(s) d s$, an integration by part (see [8]) gives that for almost any $t \in(0, T)$

$$
\begin{aligned}
\frac{1}{k} \int_{0}^{t}\left\langle\frac{\partial}{\partial t}\left[T_{s}^{\sigma}(u)-T_{s}^{\sigma}(v)\right],\right. & \left.T_{k}\left(T_{s}^{\sigma}(u)-T_{s}^{\sigma}(v)\right)\right\rangle d \tau \\
& =\frac{1}{k} \int_{\Omega} \Psi_{k}\left(T_{s}^{\sigma}(u)(t)-T_{s}^{\sigma}(v)(t)\right) d x .
\end{aligned}
$$

We deduce from the above equality that for almost any $t \in(0, T)$

$$
\begin{aligned}
\lim _{k \rightarrow 0} \lim _{\sigma \rightarrow 0} & \frac{1}{k} \int_{0}^{t}\left\langle\frac{\partial}{\partial t}\left[T_{s}^{\sigma}(u)-T_{s}^{\sigma}(v)\right], T_{k}\left(T_{s}^{\sigma}(u)-T_{s}^{\sigma}(v)\right)\right\rangle d \tau \\
& =\int_{\Omega}\left|T_{s}(u)(t)-T_{s}(v)(t)\right| d x
\end{aligned}
$$

Step 2. Reasoning as in [4] we have

$$
\begin{gathered}
\limsup _{k \rightarrow 0} \lim _{\sigma \rightarrow 0} \frac{1}{k} A_{s, k}^{\sigma}(t) \geq 0, \quad \text { for every } s>0, \\
\lim _{s \rightarrow+\infty} \lim _{k \rightarrow 0} \lim _{\sigma \rightarrow 0} \frac{1}{k} F_{s, k}^{\sigma}(t)=0,
\end{gathered}
$$

for almost any $t \in(0, T)$.

We give the argument here for completeness. Due to (4.11) and (4.10) and with the help of (2.2) we have for almost any $t \in(0, T)$

$$
\begin{aligned}
\lim _{\sigma \rightarrow 0} \frac{1}{k} A_{s, k}^{\sigma}(t)= & \frac{1}{k} \int_{0}^{t} \int_{\Omega}\left[a\left(T_{s}(u), D T_{s}(u)\right)-a\left(T_{s}(v), D T_{s}(v)\right)\right] \\
& \times D T_{k}\left(T_{s}(u)-T_{s}(v)\right) d x d \tau
\end{aligned}
$$


and which can written as

$$
\begin{aligned}
\lim _{\sigma \rightarrow 0} \frac{1}{k} A_{s, k}^{\sigma}(t)= & \frac{1}{k} \int_{0}^{t} \int_{\Omega}\left[a\left(T_{s}(u), D T_{s}(u)\right)-a\left(T_{s}(u), D T_{s}(v)\right)\right] \\
& \times D T_{k}\left(T_{s}(u)-T_{s}(v)\right) d x d \tau \\
& +\frac{1}{k} \int_{0}^{t} \int_{\Omega}\left[a\left(T_{s}(u), D T_{s}(v)\right)-a\left(T_{s}(u), D T_{s}(v)\right)\right] \\
& \times D T_{k}\left(T_{s}(u)-T_{s}(v)\right) d x d \tau .
\end{aligned}
$$

Since the operator $a$ is monotone (see (2.3)) the first term of the right hand side of (4.16) is non negative. It remains to prove that the second term goes to zero as $k$ goes to zero. Indeed using the local Lipschitz condition (3.1) on $a$ we get

$$
\begin{aligned}
\frac{1}{k} \mid & \int_{0}^{t} \int_{\Omega}\left[a\left(T_{s}(u), D T_{s}(v)\right)-a\left(T_{s}(u), D T_{s}(v)\right)\right] D T_{k}\left(T_{s}(u)-T_{s}(v)\right) d x d \tau \mid \\
\leq & \frac{1}{k} \int_{0}^{t} \int_{\Omega} \chi_{\left\{\left|T_{s}(u)-T_{s}(v)\right|<k\right\}}\left|T_{s}(u)-T_{s}(v)\right|\left(L_{s}(x, t)+\gamma_{s}\left|D T_{s}(v)\right|^{p-1}\right) \\
& \times\left|D T_{s}(u)+D T_{s}(v)\right| d x d \tau \\
\leq & \int_{\left\{0<\left|T_{s}(u)-T_{s}(v)\right|<k\right\}}\left(L_{s}(x, t)+\gamma_{s}\left|D T_{s}(v)\right|^{p-1}\right)\left|D T_{s}(u)+D T_{s}(v)\right| d x d \tau
\end{aligned}
$$

Due to the regularity of $T_{s}(u), T_{s}(v)$ and $L_{s}$ we have

$$
\left(L_{s}(x, t)+\gamma_{s}\left|D T_{s}(v)\right|^{p-1}\right)\left|D T_{s}(u)+D T_{s}(v)\right| \in L^{1}\left(Q_{T}\right) .
$$

Since $\chi_{\left\{\left|T_{s}(u)-T_{s}(v)\right|<k\right\}}$ tends to zero almost everywhere in $Q_{T}$ as $k$ goes to zero, the Lebesgue dominated convergence allows us to conclude that (4.14) holds.

As far as (4.15) is concerned we have for almost any $t \in(0, T)$

$$
\lim _{\sigma \rightarrow 0} \frac{1}{k} F_{s, k}^{\sigma}(t)=\frac{1}{k} \int_{0}^{t} \int_{\Omega} f\left(\chi_{\{|u| \leq s\}}-\chi_{\{|v| \leq s}\right) T_{k}\left(T_{s}(u)-T_{s}(v)\right) d x d \tau
$$

so that for almost any $t \in(0, T)$

$$
\lim _{k \rightarrow 0} \lim _{\sigma \rightarrow 0} \frac{1}{k} F_{s, k}^{\sigma}(t)=\int_{0}^{t} \int_{\Omega} f \times\left(\chi_{\{|u| \leq s\}}-\chi_{\{|v| \leq s}\right) \operatorname{sign}(u-v) d x d \tau,
$$

where $\operatorname{sign}(r)=r /|r|$ for any $r \neq 0$ and $\operatorname{sign}(0)=0$. Since $u$ and $v$ are finite almost everywhere in $\Omega \times(0, T)$ and since $f$ belongs to $L^{1}\left(Q_{T}\right)$ the Lebesgue dominated convergence theorem implies (4.15).

Now we claim that for almost any $t \in(0, T)$

$$
\frac{1}{k}\left(\left|\widetilde{A}_{s, k}^{\sigma}(t)\right|+\left|\widetilde{C}_{s, k}^{\sigma}(t)\right|+\left|\widetilde{G}_{s, k}^{\sigma}(t)\right|\right) \leq \frac{M_{1}}{\sigma} \Gamma(u, v, s, \sigma),
$$

where $M_{1}$ is a constant independent of $s, k$ and $\sigma$ and where $\Gamma$ is defined in Lemma 4.1 . 
Using the definition (4.8) of $T_{s}^{\sigma}$, recalling that $\nabla u=0$ almost everywhere on $\{(x, t) ; u(x, t)=r\}$ for any $r \in \mathbb{R}$ and since $a(x, t, r, \xi) \xi \geq 0$ we obtain that for any $\sigma$ and any $k>0$

$$
\begin{aligned}
\frac{1}{k}\left|\widetilde{A}_{s, k}^{\sigma}(t)\right| \leq \frac{1}{\sigma}[ & \int_{0}^{t} \int_{\Omega} \chi_{\{s<|u|<s+\sigma\}} a(u, \nabla u) \nabla u d x d \tau \\
& \left.\quad+\int_{0}^{t} \int_{\Omega} \chi_{\{s<|v|<s+\sigma\}} a(v, \nabla v) \nabla v d x d \tau\right] \\
(4.18) \leq & \frac{1}{\sigma}\left[\int_{\{s<|u|<s+\sigma\}} a(u, \nabla u) \nabla u d x d \tau+\int_{\{s<|v|<s+\sigma\}} a(v, \nabla v) \nabla v d x d \tau\right] .
\end{aligned}
$$

Similarly we have for any $\sigma$ and any $k>0$

$$
\begin{aligned}
\frac{1}{k}\left|\widetilde{C}_{s, k}^{\sigma}(t)\right| \leq \frac{1}{\sigma}\left[\int_{\{s<|u|<s+\sigma\}}|K(u)|\right. & |\nabla u| d x d \tau \\
& \left.+\int_{\{s<|v|<s+\sigma\}}|K(v)||\nabla v| d x d \tau\right] .
\end{aligned}
$$

As far as $\widetilde{G}_{s, k}(t)$ is concerned, we have for any $\sigma$ and any $k>0$

$$
\frac{1}{k}\left|\widetilde{G}_{s, k}(t)\right| \leq \frac{1}{\sigma}\left[\int_{\{s<|u|<s+\sigma\}}|g||\nabla u| d x d \tau+\int_{\{s<|v|<s+\sigma\}}|g||\nabla v| d x d \tau\right] .
$$

From assumption (2.2) together with Young inequality it follows that

$$
\begin{aligned}
\frac{1}{k}\left|\widetilde{G}_{s, k}(t)\right| \leq & \frac{M_{1}}{\sigma}\left(\int_{\{s<|u|<s+\sigma\}}\left(a(u, \nabla u) \nabla u+|g|^{p^{\prime}}\right) d x d \tau\right. \\
& \left.+\int_{\{s<|v|<s+\sigma\}}\left(a(v, \nabla v) \nabla v+|g|^{p^{\prime}}\right) d x d \tau\right),
\end{aligned}
$$

where $M_{1}$ is a generic constant depending upon $p$ and $\alpha_{0}$. Estimates (4.18)(4.20) allow us to deduce that (4.17) holds.

Now we prove that for almost any $t \in(0, T)$

$$
\limsup _{\sigma \rightarrow 0} \frac{1}{k}\left(\left|C_{s, k}^{\sigma}(t)\right|+\left|G_{s, k}^{\sigma}(t)\right|\right) \leq \frac{M_{1}}{k} \Gamma(u, v, s, k)+\omega(k),
$$

where $M_{1}$ is a constant independent of $s, k$ and $\sigma$ and where $\omega$ is a positive function such that $\lim _{k \rightarrow 0} \omega(k)=0$.

We first write that for almost any $t \in(0, T)$

$$
\begin{aligned}
\limsup _{\sigma \rightarrow 0} \frac{1}{k}\left|C_{s, k}^{\sigma}(t)\right|= & \mid \frac{1}{k} \int_{0}^{t} \int_{\Omega}\left[\chi_{\{|u| \leq s\}} K(u)-\chi_{\{|v| \leq s\}} K(v)\right] \\
& \times \nabla T_{k}\left(T_{s}(u)-T_{s}(v)\right) d x d \tau \mid \\
\leq & C_{s, k}^{1}+C_{s, k}^{2}+C_{s, k}^{3}
\end{aligned}
$$


where

$$
\begin{aligned}
& C_{s, k}^{1}=\frac{1}{k} \int_{Q_{T}} \chi_{\{|u| \leq s \wedge|v|>s\}}|K(u)|\left|\nabla T_{k}(u-s \operatorname{sign}(v))\right| d x d \tau \\
& C_{s, k}^{2}=\frac{1}{k} \int_{Q_{T}} \chi_{\{|v| \leq s \wedge|u|>s\}}|K(v)|\left|\nabla T_{k}(v-s \operatorname{sign}(u))\right| d x d \tau
\end{aligned}
$$

and

$$
C_{s, k}^{3}=\frac{1}{k} \int_{Q_{T}} \chi_{\{|v| \leq s \wedge|u| \leq s\}}|K(u)-K(v)|\left|\nabla T_{k}(u-v)\right| d x d \tau
$$

We estimate $C_{s, k}^{1}$ and $C_{s, k}^{2}$. By (2.5) we obtain

$$
\begin{aligned}
C_{s, k}^{1} & \leq \frac{1}{k} \int_{Q_{T}} \chi_{\{|u| \leq s \wedge|v|>s\}} \chi_{\{|u-s \operatorname{sign}(v)|<k\}}|K(u)||\nabla u| d x d \tau \\
& \leq \frac{1}{k} \int_{\{s-k<|u| \leq s\}}|K(u)||\nabla u| d x d \tau
\end{aligned}
$$

and similarly

$$
C_{s, k}^{2} \leq \frac{1}{k} \int_{\{s-k<|v| \leq s\}}|K(v)||\nabla v| d x d \tau .
$$

Finally, since the function $K$ is locally Lipschitz continuous, we have for some positive $L_{s}$ element of $L^{p^{\prime}}\left(Q_{T}\right)$

$$
\begin{aligned}
C_{s, k}^{3} & =\frac{1}{k} \int_{Q_{T}} \chi_{\{|v| \leq s \wedge|u| \leq s\}}|K(u)-K(v)|\left|\nabla T_{k}\left(T_{s}(u)-T_{s}(v)\right)\right| d x d \tau \\
\leq & \frac{1}{k} \int_{Q_{T}} \chi_{\left\{0<\left|T_{s}(v)-T_{s}(u)\right|<k\right\}} L_{s}(x, \tau)\left|T_{s}(u)-T_{s}(v)\right| \\
& \times\left|\nabla T_{k}\left(T_{s}(u)-T_{s}(v)\right)\right| d x d \tau \\
& \leq \int_{Q_{T}} \chi_{\left\{0<\left|T_{s}(v)-T_{s}(u)\right|<k\right\}} L_{s}(x, \tau)\left|\nabla T_{k}\left(T_{s}(u)-T_{s}(v)\right)\right| d x d \tau \\
& \leq \int_{Q_{T}} \chi_{\left\{0<\left|T_{s}(v)-T_{s}(u)\right|<k\right\}} L_{s}(x, \tau)\left(\left|\nabla T_{s}(u)\right|+\left|\nabla T_{s}(v)\right|\right) d x d \tau .
\end{aligned}
$$

Since $L_{s}$ belongs to $L^{p^{\prime}}\left(Q_{T}\right)$ and due to (2.13) the function $L_{s}(x, \tau)\left(\left|\nabla T_{s}(u)\right|+\right.$ $\left.\left|\nabla T_{s}(v)\right|\right)$ belongs to $L^{1}\left(Q_{T}\right)$. Because $\chi_{\left\{0<\left|T_{s}(v)-T_{s}(u)\right|<k\right\}}$ tends to 0 almost everywhere in $Q_{T}$ as $k$ goes to 0 and is bounded by 1 , the Lebesgue dominated convergence theorem leads to

$$
\lim _{k \rightarrow 0} C_{s, k}^{3}=0, \quad \text { for any } s>0 .
$$


In order to estimate $G_{s, k}(t)$, we obtain for almost any $t \in(0, T)$

$$
\begin{aligned}
\limsup _{\sigma \rightarrow 0} \frac{1}{k}\left|G_{s, k}^{\sigma}(t)\right|= & \mid \frac{1}{k} \int_{0}^{t} \int_{\Omega}\left[\chi_{\{|u| \leq s\}} g-\chi_{\{|v| \leq s\}} g\right] \\
& \times \nabla T_{k}\left(T_{s}(u)-T_{s}(v)\right) d x d \tau \mid \\
\leq & G_{s, k}^{1}+G_{s, k}^{2},
\end{aligned}
$$

where

$$
G_{s, k}^{1}=\frac{1}{k} \int_{Q_{T}} \chi_{\{|u| \leq s \wedge|v|>s\}}|g|\left|\nabla T_{k}(u-s \operatorname{sign}(v))\right| d x d \tau
$$

and

$$
G_{s, k}^{2}=\frac{1}{k} \int_{Q_{T}} \chi_{\{|v| \leq s \wedge|u|>s\}}|g|\left|\nabla T_{k}(v-s \operatorname{sign}(u))\right| d x d \tau .
$$

Since we have

$$
G_{s, k}^{1} \leq \frac{1}{k} \int_{\{s-k<|u| \leq s\}}|g||\nabla u| d x d \tau
$$

similar arguments to the ones used to deal with $\widetilde{G}_{s, k}$ yield that

$$
G_{s, k}^{1} \leq \frac{M_{1}}{k} \int_{\{s-k<|u|<s\}}\left(a(u, \nabla u) \nabla u+|g|^{p^{\prime}}\right) d x d \tau,
$$

where $M$ is a constant depending upon $p$ and $\alpha_{0}$. With $v$ in place of $u$ in $G_{s, k}^{2}$ we also have

$$
G_{s, k}^{2} \leq \frac{M_{1}}{k} \int_{\{s-k<|v|<s\}}\left(a(v, \nabla v) \nabla v+|g|^{p^{\prime}}\right) d x d \tau .
$$

Estimates (4.22)-(4.26) imply (4.21)

Step 3. We are now in a position to prove that $u=v$ almost everywhere in $Q_{T}$. Passing to the limit-sup as $\sigma$ goes to 0 and then to the limit-sup as $k$ goes to zero in (4.9) with the help of (4.13), (4.14), (4.15), (4.17) and (4.21) leads to for any $s>0$ and for almost any $t \in(0, T)$

$$
\begin{aligned}
\int_{\Omega}\left|T_{s}(u)(t)-T_{s}(v)(t)\right| d x \leq & M_{1} \limsup _{k \rightarrow 0} \frac{1}{k} \Gamma(u, v, s, k) \\
& +M_{1} \limsup _{\sigma \rightarrow 0} \frac{1}{\sigma} \Gamma(u, v, s, \sigma)+\omega(s) .
\end{aligned}
$$

Recalling that $u$ (resp. $v$ ) is finite almost everywhere in $Q_{T}, T_{s}(u)(t)$ (resp. $T_{s}(v)(t)$ ) converges almost everywhere to $u(t)$ (resp. $v(t)$ ) as $s$ goes to infinity for almost any $t \in(0, T)$. By Fatou lemma we can pass to the limit-inf as $s$ goes to $+\infty$ in (4.27) and we obtain for almost any $t \in(0, T)$

$$
\int_{\Omega}|u(t)-v(t)| d x \leq 2 M_{1} \liminf _{s \rightarrow+\infty} \limsup _{k \rightarrow 0} \frac{1}{k} \Gamma(u, v, s, k) .
$$


Lemma 4.1 allows us to conclude that $\int_{\Omega}|u(t)-v(t)| d x=0$ for almost any $t \in(0, T)$ so that $u=v$ almost everywhere in $Q_{T}$.

In the case of the complete operator we need the following lemma which concerns Boccardo-Gallouët kind estimates in Lorentz spaces.

Lemma 4.2. Assume that $Q_{T}=\Omega \times(0, T)$ with $\Omega$ open subset of $\mathbb{R}^{N}$ of finite measure and $p>1$. Let be $u$ a measurable function satisfying

$T_{k}(u) \in L^{\infty}\left((0, T) ; L^{2}(\Omega)\right) \cap L^{p}\left((0, T) ; W_{0}^{1, p}(\Omega)\right)$ for every $k>0$ and such that for $\alpha>\frac{2(N+1)}{N+2}$

$$
\sup _{t \in(0, T)} \int_{\Omega}\left|T_{k}(u(t))\right|^{2} \leq k M \text { and } \int_{0}^{T} \int_{\Omega}\left|\nabla T_{k}(u)\right|^{\alpha} \leq C_{0} k^{\frac{\alpha}{2}} M^{\frac{\alpha}{2}},
$$

where $M$ and $C_{0}$ are positive constant. Then

$$
\|u\|_{L}^{\frac{\alpha(N+2)}{2 N}, \infty}{ }_{\left(Q_{T}\right)} \leq C M
$$

and

$$
\|\mid \nabla u\|_{L^{\frac{\alpha(N+2)}{2(N+1)}, \infty}\left(Q_{T}\right)} \leq C M,
$$

where $C$ is a constant depending only on $N$ and $C_{0}$.

Such a result being standard we omit the proof of Lemma 4.2 (see for example the proof of Lemma A.1 given in [12 with a very few modifications).

Proof of Theorem 3.2. The proof is divided into four steps. As in the previous theorem we consider two renormalized solutions $u$ and $v$ of the Problem (2.1) for the same data $f, g$ and $u_{0}$. In Step 1, we plug the test function $T_{k}\left(T_{s}^{\sigma}(u)-T_{s}^{\sigma}(v)\right)$ in the difference of the equations (2.15) for $u$ and $v$ with $S=T_{s}^{\sigma}$ (defined in (4.8)) and we obtain equation (4.32). Step 2 is devoted to estimate the terms of (4.32). In Step 3 we pass to the limit as $\sigma \rightarrow 0$ and $s \rightarrow+\infty, k$ being fixed. Finally in Step 4 using Lemma 4.2 we give an estimate of $\nabla u-\nabla v$ in some suitable Lorentz spaces, which allows us to conclude that $u=v$.

Step 1. Let $u$ and $v$ be two renormalized solutions to Problem (2.1) for the same data $f, g$ and $u_{0}$. For every real number $s>0$ and $\sigma>0$ we take $S=T_{s}^{\sigma}$ in (2.15) for $u$ and $v$. Subtracting these two equations and plugging the test function $\frac{1}{k} T_{k}\left(T_{s}^{\sigma}(u)-T_{s}^{\sigma}(v)\right)$, we obtain upon integration on $(0, t)$, that

$$
\begin{aligned}
\int_{0}^{t}\left\langle\frac{\partial}{\partial t}[\right. & \left.\left.T_{s}^{\sigma}(u)-T_{s}^{\sigma}(v)\right], T_{k}\left(T_{s}^{\sigma}(u)-T_{s}^{\sigma}(v)\right)\right\rangle d \tau \\
& +A_{s, k}^{\sigma}(t)+\widetilde{A}_{s, k}^{\sigma}(t) \\
=B_{s, k}^{\sigma}(t)+ & C_{s, k}^{\sigma}(t)+\widetilde{C}_{s, k}^{\sigma}(t)+F_{s, k}^{\sigma}(t)+G_{s, k}^{\sigma}(t)+\widetilde{G}_{s, k}^{\sigma}(t)
\end{aligned}
$$


for every $k>0, s>0, \sigma>0$ and for almost any $t \in(0, T)$, where

$$
\begin{aligned}
B_{s, k}^{\sigma}(t)=- & \int_{0}^{t} \int_{\Omega}\left[\left(T_{s}^{\sigma}\right)^{\prime}(u) H(\nabla u)-\left(T_{s}^{\sigma}\right)^{\prime}(v) H(\nabla u)\right] \\
& \times T_{k}\left(T_{s}^{\sigma}(u)-T_{s}^{\sigma}(v)\right) d x d \tau
\end{aligned}
$$

and the remained terms are defined in the proof of Theorem 3.1. We now pass to the limit in (4.32) as $\sigma$ goes to zero and then as $s$ goes to $+\infty$.

Step 2. We recall that for almost any $t \in(0, T)$

$$
\begin{aligned}
\int_{0}^{t}\left\langle\frac{\partial}{\partial t}\left[T_{s}^{\sigma}(u)-T_{s}^{\sigma}(v)\right], T_{k}\left(T_{s}^{\sigma}(u)\right.\right. & \left.\left.-T_{s}^{\sigma}(v)\right)\right\rangle d \tau \\
& =\int_{\Omega} \Psi_{k}\left(T_{s}^{\sigma}(u)(t)-T_{s}^{\sigma}(v)(t)\right) d x .
\end{aligned}
$$

Due to the definition of $T_{s}^{\sigma}$ we obtain that

$$
\begin{aligned}
\lim _{\sigma \rightarrow 0} \int_{0}^{t}\left\langle\frac{\partial}{\partial t}\left[T_{s}^{\sigma}(u)-T_{s}^{\sigma}(v)\right],\right. & \left.T_{k}\left(T_{s}^{\sigma}(u)-T_{s}^{\sigma}(v)\right)\right\rangle d \tau \\
& =\int_{\Omega} \Psi_{k}\left(T_{s}(u)(t)-T_{s}(v)(t)\right) d x
\end{aligned}
$$

and since $u$ and $v$ are finite almost everywhere in $Q_{T}$, from Fatou lemma it follows that

$$
\begin{aligned}
\liminf _{s \rightarrow+\infty} \lim _{\sigma \rightarrow 0} \int_{0}^{t}\left\langle\frac{\partial}{\partial t}\left[T_{s}^{\sigma}(u)-T_{s}^{\sigma}(v)\right],\right. & \left.T_{k}\left(T_{s}^{\sigma}(u)-T_{s}^{\sigma}(v)\right)\right\rangle d \tau \\
& \geq \int_{\Omega} \Psi_{k}(u(t)-v(t)) d x
\end{aligned}
$$

Since $H(\nabla u)$ and $H(\nabla v)$ belong to $L^{1}\left(Q_{T}\right)$ and since $u$ and $v$ are finite almost everywhere in $Q_{T}$, the Lebesgue theorem yields that

$$
\lim _{s \rightarrow+\infty} \lim _{\sigma \rightarrow 0} B_{s, k}^{\sigma}(t)=-\int_{0}^{t} \int_{\Omega}[H(\nabla u)-H(\nabla v)] T_{k}(u-v) d x d \tau .
$$

Using the Lipschitz condition (3.4) on $H$ and (3.6) we obtain

$$
\begin{gathered}
\int_{0}^{t} \int_{\Omega}\left|[H(\nabla u)-H(\nabla v)] T_{k}(u-v)\right| d x d \tau \leq \\
k\|b\|_{L^{\lambda, 1}\left(Q_{t}\right)}\|1+|\nabla u|+|\nabla v|\|_{L^{q, \infty}\left(Q_{t}\right)}^{\sigma}\||\nabla u-\nabla v|\|_{L^{\theta, \infty}\left(Q_{t}\right)}
\end{gathered}
$$

with

$$
\frac{1}{\lambda}+\frac{\sigma}{q}+\frac{1}{\theta}=1, \quad 1 \leq q \leq \frac{N(p-1)+p}{N+1}, \quad \theta=\frac{N+2}{N+1} \quad \text { and } \quad \lambda \geq N+2 .
$$

It follows that for almost any $t \in(0, T)$

$$
\begin{aligned}
& \lim _{s \rightarrow+\infty} \lim _{\sigma \rightarrow 0}\left|B_{s, k}^{\sigma}(t)\right| \\
& \quad \leq k\|b\|_{L^{\lambda, 1}\left(Q_{t}\right)}\|1+|\nabla u|+|\nabla v|\|_{L^{q, \infty}\left(Q_{t}\right)}^{\sigma}\||\nabla u-\nabla v|\|_{L^{\theta, \infty}\left(Q_{t}\right)} .
\end{aligned}
$$


Since $f$ belongs to $L^{1}\left(Q_{T}\right)$ while $u$ and $v$ are finite almost everywhere in $Q_{T}$ we have

$$
\begin{aligned}
\lim _{s \rightarrow+\infty} \lim _{\sigma \rightarrow 0} F_{s, k}^{\sigma}(t)=\lim _{s \rightarrow+\infty} \int_{0}^{t} \int_{\Omega} f\left[\chi_{\{|u| \leq s\}}-\chi_{\{|v| \leq s\}}\right] \\
\times T_{k}\left(T_{s}(u)-T_{s}(v)\right) d x d \tau=0
\end{aligned}
$$

We now deal with $A_{s, k}^{\sigma}, C_{s, k}^{\sigma}$ and $G_{s, k}^{\sigma}$. From the definition of $T_{s}^{\sigma}$ and (3.5) we get

$$
\begin{aligned}
& \lim _{\sigma \rightarrow 0} A_{s, k}^{\sigma}(t)= \int_{0}^{t} \int_{\Omega}\left[\chi_{\{|u| \leq s\}} a(\nabla u)-\chi_{\{|v| \leq s\}} a(\nabla v)\right] \\
& \times \nabla T_{k}\left(T_{s}(u)-T_{s}(v)\right) d x d \tau \\
&= \int_{0}^{t} \int_{\Omega} \chi_{\left\{T_{s}(u)-T_{s}(v) \mid<k\right\}}\left[a\left(\nabla T_{s}(u)\right)-a\left(\nabla T_{s}(v)\right)\right] \\
& \times\left(\nabla T_{s}(u)-\nabla T_{s}(v)\right) d x d \tau \\
& \geq \beta \int_{0}^{t} \int_{\Omega} \chi_{\left\{T_{s}(u)-T_{s}(v) \mid<k\right\}}\left(1+\left|\nabla T_{s}(u)\right|+\left|\nabla T_{s}(v)\right|\right)^{p-2} \\
& \times\left|\nabla T_{s}(u)-\nabla T_{s}(v)\right|^{2} d x d \tau .
\end{aligned}
$$

Since $u$ and $v$ are finite almost everywhere Fatou lemma then implies

$$
\begin{aligned}
\liminf _{s \rightarrow+\infty} \lim _{\sigma \rightarrow 0} A_{s, k}^{\sigma} \geq & \beta \int_{0}^{t} \int_{\Omega} \chi_{\{|u-v|<k\}}(1+|\nabla u|+|\nabla(v)|)^{p-2} \\
& \times|\nabla u-\nabla v|^{2} d x d \tau .
\end{aligned}
$$

Using assumption (3.3) we have

$$
\begin{aligned}
\lim _{\sigma \rightarrow 0}\left|C_{s, k}^{\sigma}(t)\right| \leq & \int_{0}^{t} \int_{\Omega}\left|\chi_{\{|u| \leq s\}} K(u)-\chi_{\{|v| \leq s\}} K(v)\right| \\
\times\left|\nabla T_{k}\left(T_{s}(u)-T_{s}(v)\right)\right| d x d \tau & \\
\leq & \int_{0}^{t} \int_{\Omega} \chi_{\{|u| \leq s \wedge|v| \leq s\}} c(x, \tau)(1+|u|+|v|)^{\tau} \\
& +\int_{0}^{t} \int_{\Omega} \chi_{\{s-k<|v| \leq s\}}|K(v)||\nabla v| d x d \tau \\
& +\int_{0}^{t} \int_{\Omega} \chi_{\{s-k<|u| \leq s\}}|K(u)||\nabla u| d x d \tau
\end{aligned}
$$

From Hölder inequality and condition (3.6) we obtain

$$
\begin{aligned}
\int_{0}^{t} \int_{\Omega} c(x, \tau)(1+|u|+|v|)^{\tau}|\nabla u-\nabla v| d x d \tau \\
\quad \leq\|c\|_{L^{r, 1}\left(Q_{t}\right)}\|1+|u|+|v|\|_{L^{q, \infty}\left(Q_{t}\right)}^{\tau}\||\nabla u-\nabla v|\|_{L^{\theta, \infty}\left(Q_{t}\right)}
\end{aligned}
$$


with

$$
\frac{1}{r}+\frac{\tau}{\bar{q}}+\frac{1}{\theta}=1, \quad 1 \leq \bar{q} \leq \frac{N(p-1)+p}{N}, \quad \theta=\frac{N+2}{N+1} \quad \text { and } \quad r>\frac{N+p}{p-1} .
$$

From the regularities of $c, u, v, \nabla u$ and $\nabla v$ it follows that $c(x, \tau)(1+|u|+$ $|v|)^{\tau}|\nabla u-\nabla v|$ belongs to $L^{1}\left(Q_{t}\right)$ for any $t \in(0, T)$. Recalling the definition (4.2) of $\Theta$ in Lemma 4.1 leads to

$$
\begin{aligned}
\lim _{\sigma \rightarrow 0}\left|C_{s, k}^{\sigma}(t)\right| \leq k\|c\|_{L^{r, 1}\left(Q_{t}\right)}\|1+|u|+|v|\|_{L^{\bar{q}, \infty}\left(Q_{t}\right)}^{\tau} \\
\times\||\nabla u-\nabla v|\|_{L^{\theta, \infty}\left(Q_{t}\right)}+\Theta(u, v, s, k)
\end{aligned}
$$

for any $k>0$.

We now study $G_{s, k}^{\sigma}(t)$. We first have

$$
\lim _{\sigma \rightarrow 0} G_{s, k}^{\sigma}(t)=\int_{0}^{t} \int_{\Omega} g\left[\chi_{\{|u|<s\}}-\chi_{\{|v|<s\}}\right] \nabla T_{k}\left(T_{s}(u)-T_{s}(v)\right) d x d t .
$$

It follows that

$$
\begin{aligned}
\lim _{\sigma \rightarrow 0}\left|G_{s, k}^{\sigma}(t)\right| \leq & \int_{0}^{t} \int_{\Omega} \chi_{\{s-k<|u|<s\}}|g||\nabla u| d x d \tau \\
& +\int_{0}^{t} \int_{\Omega} \chi_{\{s-k<|v|<s\}}|g||\nabla v| d x d \tau .
\end{aligned}
$$

With Young inequality and integrating on $Q_{T}$ in place of $\Omega \times(0, t)$ we obtain

$$
\begin{aligned}
\lim _{\sigma \rightarrow 0}\left|G_{s, k}^{\sigma}(t)\right| \leq & \frac{1}{p^{\prime}} \int_{Q_{T}}\left(\chi_{\{s-k<|u|<s\}}+\chi_{\{s-k<|v|<s\}}\right)|g|^{p^{\prime}} d x d \tau \\
& +\frac{1}{p} \int_{\{s-k<|u|<s\}}|\nabla u|^{p} d x d \tau+\frac{1}{p} \int_{\{s-k<|v|<s\}}|\nabla v|^{p} d x d \tau .
\end{aligned}
$$

Since $u$ and $v$ are finite almost everywhere in $Q_{T}$ the function $\left(\chi_{\{s-k<|u|<s\}}+\right.$ $\left.\chi_{\{s-k<|v|<s\}}\right)|g|^{p^{\prime}}$ converges to zero as $s$ goes to $+\infty$ in $L^{1}\left(Q_{T}\right)$. Since the operator $a$ is elliptic (see assumption (2.2) ) and recalling the definition of $\Theta$ in Lemma 4.1 we then obtain

$$
\lim _{\sigma \rightarrow 0}\left|G_{s, k}^{\sigma}(t)\right| \leq \frac{1}{\alpha_{0}} \Theta(u, v, s, k)+\omega(s)
$$

where $\omega(s)$ is a generic function which converges to 0 as $s$ goes to infinity.

We recall (see (4.17) in the proof of Theorem 3.1) that for almost any $t \in(0, T)$

$$
\left|\widetilde{A}_{s, k}^{\sigma}(t)\right|+\left|\widetilde{C}_{s, k}^{\sigma}(t)\right|+\left|\widetilde{G}_{s, k}^{\sigma}(t)\right| \leq \frac{M_{1} k}{\sigma} \Gamma(u, v, s, \sigma) .
$$


From estimates (4.40), (4.41) and (4.42) it follows that

$$
\begin{aligned}
\limsup _{\sigma \rightarrow 0}( & \left.\left|C_{s, k}^{\sigma}(t)\right|+\left|\widetilde{A}_{s, k}^{\sigma}(t)\right|+\left|G_{s, k}^{\sigma}(t)\right|+\left|\widetilde{C}_{s, k}^{\sigma}(t)\right|+\left|\widetilde{G}_{s, k}^{\sigma}(t)\right|\right) \\
\leq & k\|c\|_{L^{r, 1}\left(Q_{t}\right)}\|1+|u|+|v|\|_{L^{\bar{q}, \infty}\left(Q_{t}\right)}^{\tau}\||\nabla u-\nabla v|\|_{L^{\theta, \infty}\left(Q_{t}\right)} \\
& +\Theta(u, v, s, k)+\frac{1}{\alpha_{0}} \Theta(u, v, s, k)+\omega(s) \\
& +M_{1} k \limsup _{\sigma \rightarrow 0} \frac{1}{\sigma} \Gamma(u, v, s, \sigma) .
\end{aligned}
$$

By the above inequality and Lemma 4.1 we can conclude that for almost any $t \in(0, T)$

$$
\begin{aligned}
& \liminf _{s \rightarrow+\infty} \limsup _{\sigma \rightarrow 0}\left(\left|C_{s, k}^{\sigma}(t)\right|+\left|\widetilde{A}_{s, k}^{\sigma}(t)\right|+\left|G_{s, k}^{\sigma}(t)\right|+\left|\widetilde{C}_{s, k}^{\sigma}(t)\right|+\left|\widetilde{G}_{s, k}^{\sigma}(t)\right|\right) \\
& \leq k\|c\|_{L^{r, 1}\left(Q_{t}\right)}\|1+|u|+|v|\|_{L^{q, \infty}\left(Q_{t}\right)}^{\tau}\||\nabla u-\nabla v|\|_{L^{\theta, \infty}\left(Q_{t}\right)} .
\end{aligned}
$$

Step 3. We are now able to pass to the limit in (4.32). Indeed gathering (4.33), (4.34), (4.35), (4.37) and (4.43), we get

$$
\begin{aligned}
\int_{\Omega} \Psi_{k} & (u(t)-v(t)) d x \\
& +\frac{\beta}{2} \int_{0}^{t} \int_{\Omega} \chi_{\{|u-v|<k\}}(1+|\nabla u|+|\nabla(v)|)^{p-2}|\nabla u-\nabla v|^{2} d x d \tau \\
\leq & k\|c\|_{L^{r, 1}\left(Q_{t}\right)}\|1+|u|+|v|\|_{L^{\bar{q}, \infty}\left(Q_{t}\right)}^{\tau}\||\nabla u-\nabla v|\|_{L^{\theta, \infty}\left(Q_{t}\right)} \\
& +k\|b\|_{L^{\lambda, 1}\left(Q_{t}\right)}\|1+|\nabla u|+|\nabla v|\|_{L^{q, \infty}\left(Q_{t}\right)}^{\sigma}\||\nabla u-\nabla v|\|_{L^{\theta, \infty}\left(Q_{t}\right)}
\end{aligned}
$$

for almost any $t \in(0, T)$. It is worth noting that the above inequality implies

$$
\chi_{\{|u-v|<k\}}(1+|\nabla u|+|\nabla(v)|)^{p-2}|\nabla u-\nabla v|^{2} \in L^{1}\left(Q_{T}\right) .
$$

Since $\left(1+|\xi|+\left|\xi^{\prime}\right|\right)^{p-2}\left|\xi-\xi^{\prime}\right|^{2} \geq\left|\xi-\xi^{\prime}\right|^{2}$ for any $\xi, \xi^{\prime}$ in $\mathbb{R}^{N}$, we obtain that $T_{k}(u-v)$ belongs to $L^{2}\left((0, T) ; H_{0}^{1}(\Omega)\right)$.

Due to the definition of $\Psi_{k}$, taking the supremum for $t \in\left(0, t_{1}\right)$, where $t_{1} \in(0, T)$ will be chosen later, leads to

$$
\frac{1}{2} \sup _{t \in\left(0, t_{1}\right)} \int_{\Omega}\left|T_{k}(u-v)\right|^{2} d x+\frac{\beta}{2} \int_{0}^{t_{1}} \int_{\Omega}\left|\nabla T_{k}(u-v)\right|^{2} d x d \tau \leq k M
$$

where

$$
\begin{aligned}
M= & \|b\|_{L^{\lambda, 1}\left(Q_{t_{1}}\right)}\|1+|\nabla u|+|\nabla v|\|_{L^{q, \infty}\left(Q_{t_{1}}\right)}^{\sigma}\||\nabla u-\nabla v|\|_{L^{\theta, \infty}\left(Q_{t_{1}}\right)} \\
& +\|c\|_{L^{r, 1}\left(Q_{t_{1}}\right)}\|1+|u|+|v|\|_{L^{\bar{q}, \infty}\left(Q_{t_{1}}\right)}^{\tau}\||\nabla u-\nabla v|\|_{L^{\theta, \infty}\left(Q_{t_{1}}\right)} .
\end{aligned}
$$

By (4.44) and Lemma 4.2 we get

$$
\|\nabla u-\nabla v\|_{L^{\theta, \infty}\left(Q_{t_{1}}\right)} \leq C M
$$

for some constant $C>0$ independent on $u$ and $v$ and $\theta=\frac{N+2}{N+1}$. 
Step 4. Using (4.45) and (4.46) we obtain

$$
\begin{aligned}
\||\nabla u-\nabla v|\|_{L^{\theta, \infty}\left(Q_{t_{1}}\right) \leq C} & {\left[\|b\|_{L^{\lambda, 1}\left(Q_{t_{1}}\right)}\|1+|\nabla u|+|\nabla v|\|_{L^{q, \infty}\left(Q_{t_{1}}\right)}^{\sigma}\right.} \\
& \left.+\|c\|_{L^{r, 1}\left(Q_{t_{1}}\right)}\|1+|u|+|v|\|_{L^{\bar{q}, \infty}\left(Q_{t_{1}}\right)}^{\tau}\right] \\
\times & \||\nabla u-\nabla v|\|_{L^{\theta, \infty}\left(Q_{t_{1}}\right)} .
\end{aligned}
$$

Since $c$ belongs to $L^{r, 1}\left(Q_{T}\right)$ and since $b$ belongs to $L^{\lambda, 1}\left(Q_{T}\right)$, choosing $t_{1}$ small enough such that

$$
\begin{aligned}
1-C\left(\|b\|_{L^{\lambda, 1}\left(Q_{t_{1}}\right)} \| 1\right. & +|\nabla u|+|\nabla v| \|_{L^{q, \infty}\left(Q_{t_{1}}\right)}^{\sigma} \\
& \left.+\|c\|_{L^{r, 1}\left(Q_{t_{1}}\right)}\|1+|u|+|v|\|_{L^{\bar{q}, \infty}\left(Q_{t_{1}}\right)}^{\tau}\right)>0,
\end{aligned}
$$

then (4.47) gives

$$
\||\nabla u-\nabla v|\|_{L^{\theta, \infty}\left(Q_{t_{1}}\right)} \leq 0
$$

with $\theta=\frac{N+2}{N+1}$.

Now we use the same technique as in 22, (see also [12]). We consider a partition of the entire interval $[0, T]$ into a finite number of intervals $\left[0, t_{1}\right]$, $\left[t_{1}, t_{2}\right], \ldots,\left[t_{n-1}, T\right]$ such that for each interval $\left[t_{i-1}, t_{i}\right]$ a similar condition to (4.48) holds. In this way in each cylinder $Q_{t_{i}}=\Omega \times\left[t_{i-1}, t_{i}\right]$ we obtain estimates of type (4.49). Then we can deduce that

$$
\||\nabla u-\nabla v|\|_{L^{\theta, \infty}\left(Q_{T}\right)} \leq 0 \quad \text { for some } \theta \geq 1,
$$

that implies that $u=v$ a.e. in $Q_{T}$.

Proof of Theorem 3.3. The strategy of the proof is the same as in Theorem 3.2 and relies on passing to the limit in (4.32). The main differences are in dealing the terms $A_{s, k}^{\sigma}(t), B_{s, k}^{\sigma}$ and $C_{s, k}^{\sigma}(t)$ and the estimate on $\nabla T_{k}(u-v)$. We recall (4.32):

$$
\begin{aligned}
\int_{0}^{t}\left\langle\frac{\partial}{\partial t}\left[T_{s}^{\sigma}(u)-T_{s}^{\sigma}(v)\right],\right. & \left.T_{k}\left(T_{s}^{\sigma}(u)-T_{s}^{\sigma}(v)\right)\right\rangle d \tau \\
& +A_{s, k}^{\sigma}(t)+\widetilde{A}_{s, k}^{\sigma}(t) \\
=B_{s, k}^{\sigma}(t)+ & C_{s, k}^{\sigma}(t)+\widetilde{C}_{s, k}^{\sigma}(t)+F_{s, k}^{\sigma}(t)+G_{s, k}^{\sigma}(t)+\widetilde{G}_{s, k}^{\sigma}(t)
\end{aligned}
$$

for any $s>0$, any $k>0$ and any $\sigma>0$ and for almost any $t \in(0, T)$. Reasoning as in the previous theorem by assumption (3.7), we obtain that

$$
\liminf _{s \rightarrow+\infty} \lim _{\sigma \rightarrow 0} A_{s, k}^{\sigma}(t) \geq \beta \int_{0}^{t} \int_{\Omega} \chi_{\{|u-v|<k\}} \frac{\mid \nabla u-\nabla v)\left.\right|^{2}}{(|\nabla u|+|\nabla v|)^{2-p}} d x d \tau .
$$


As far as $B_{s, k}^{\sigma}(t)$ is concerned, a few computations, estimates (2.18) and (2.19), condition (3.8) and Hölder inequality lead to

$$
\begin{gathered}
\int_{0}^{t} \int_{\Omega}\left|[H(\nabla u)-H(\nabla v)] T_{k}(u-v)\right| d x d \tau \leq \\
k\|b\|_{L^{\lambda, 1}\left(Q_{t}\right)}\|1+|\nabla u|+|\nabla v|\|_{L^{q, \infty}\left(Q_{t}\right)}^{\sigma}\||\nabla u-\nabla v|\|_{L^{\theta, \infty}\left(Q_{t}\right)}
\end{gathered}
$$

with

$$
\begin{gathered}
\frac{1}{\lambda}+\frac{\sigma}{q}+\frac{1}{\theta}=1, \quad 1 \leq q \leq \frac{N(p-1)+p}{N+1} \\
\theta=\frac{\alpha(N+2)}{2(N+1)}, \quad \lambda \geq N+2 \quad \text { and } \quad \alpha<\frac{2 p(N+1)-2 N}{N+2} .
\end{gathered}
$$

Similarly we obtain

$$
\begin{aligned}
& \liminf _{s \rightarrow+\infty} \lim _{\sigma \rightarrow 0}\left|C_{s, k}^{\sigma}(t)\right| \leq k\|c\|_{L^{r, 1}\left(Q_{t}\right)}\|1+|u|+|v|\|_{L^{\bar{q}, \infty}\left(Q_{t}\right)}^{\tau} \\
& \times\||\nabla u-\nabla v|\|_{L^{\theta, \infty}\left(Q_{t}\right)}
\end{aligned}
$$

with

$$
\begin{gathered}
\frac{1}{r}+\frac{\tau}{\bar{q}}+\frac{1}{\theta}=1, \quad 1 \leq \bar{q} \leq \frac{N(p-1)+p}{N} \\
\theta=\frac{\alpha(N+2)}{2(N+1)}, \quad r>\frac{N+p}{p-1}, \quad \text { and } \quad \alpha<\frac{2 p(N+1)-2 N}{N+2} .
\end{gathered}
$$

Then the analogous of (4.44) is

$$
\frac{1}{2} \sup _{t \in\left(0, t_{1}\right)} \int_{\Omega}\left|T_{k}(u-v)\right|^{2} d x+\beta \int_{0}^{t_{1}} \int_{\Omega} \frac{\left|\nabla T_{k}(u-v)\right|^{2}}{(|\nabla u|+|\nabla v|)^{2-p}} d x d t \leq k M
$$

where $t_{1}$ will be chosen later and $M$ is defined in the proof of Theorem 3.2 (see (4.45)). Then we obtain that

$$
\begin{gathered}
\beta \int_{0}^{t_{1}} \int_{\Omega} \frac{\left|\nabla T_{k}(u-v)\right|^{2}}{(|\nabla u|+|\nabla v|)^{2-p}} d x d t \leq M k, \\
\frac{1}{2} \sup _{t \in\left(0, t_{1}\right)} \int_{\Omega}\left|T_{k}(u-v)\right|^{2} \leq M k, .
\end{gathered}
$$


If $1 \leq \alpha<p$, by Hölder inequality and (4.53) we have

$$
\begin{aligned}
\int_{0}^{t_{1}} \int_{\Omega}\left|\nabla T_{k}(u-v)\right|^{\alpha} d x d t \\
=\int_{0}^{t_{1}} \int_{\Omega}\left|\nabla T_{k}(u-v)\right|^{\alpha} \frac{(|\nabla u|+|\nabla v|)^{(2-p) \frac{\alpha}{2}}}{(|\nabla u|+|\nabla v|)^{(2-p) \frac{\alpha}{2}}} d x d t \\
\leq\left(\int_{0}^{t_{1}} \int_{\Omega} \frac{\left|\nabla T_{k}(u-v)\right|^{2}}{(|\nabla u|+|\nabla v|)^{2-p}} d x d t\right)^{\frac{\alpha}{2}} \\
\quad \times\left(\int_{0}^{t_{1}} \int_{\Omega}(|\nabla u|+|\nabla v|)^{\frac{(2-p) \alpha}{2-\alpha}} d x d t\right)^{\frac{2-\alpha}{2}} \\
\leq(M k)^{\frac{\alpha}{2}}\left(\int_{0}^{t_{1}} \int_{\Omega}(|\nabla u|+|\nabla v|)^{\frac{(2-p) \alpha}{2-\alpha}} d x d t\right)^{\frac{2-\alpha}{2}} .
\end{aligned}
$$

By (2.18) the last integral in (4.55) is finite if

$$
\alpha<\frac{2 p(N+1)-2 N}{N+2} \text {. }
$$

We observe that condition (4.56) and the conditon on $\alpha$ in Lemma 4.2 are compatible only if $p>2-\frac{1}{N+1}$. Then by (4.55), (4.56) and by Hölder inequality we have

$$
\int_{0}^{t_{1}} \int_{\Omega}\left|\nabla T_{k}(u-v)\right|^{\alpha} d x d t \leq C(M k)^{\frac{\alpha}{2}}
$$

where $C$ is constant independent on $t_{1}$.

By (4.54), (4.57) and the definition of $M$ Lemma 4.2 gives for $\theta=\frac{\alpha(N+2)}{2(N+1)}$

$$
\begin{aligned}
\||\nabla u-\nabla v|\|_{L^{\theta, \infty}\left(Q_{t_{1}}\right)} \leq C & {\left[\|b\|_{L^{\lambda, 1}\left(Q_{t_{1}}\right)}\|1+|\nabla u|+|\nabla v|\|_{L^{q, \infty}\left(Q_{t_{1}}\right)}^{\sigma}\right.} \\
& \left.+\|c\|_{L^{r, 1}\left(Q_{t_{1}}\right)}\|1+|u|+|v|\|_{L^{\bar{q}, \infty}\left(Q_{t_{1}}\right)}^{\tau}\right] \\
& \times\||\nabla u-\nabla v|\|_{L^{\theta, \infty}\left(Q_{t_{1}}\right)} .
\end{aligned}
$$

Under hypotheses (3.8) we can choose $t_{1}$ small enough such that (4.48) holds. Then by (4.58) and (4.48) it follows that for $\theta=\frac{\alpha(N+2)}{2(N+1)}$

$$
\||\nabla u-\nabla v|\|_{L^{\theta, \infty}\left(Q_{t_{1}}\right)} \leq 0 .
$$

Arguing as in Theorem 3.2 we conclude that $u=v$ almost everywhere in $Q_{T}$.

\section{ACKNowledgement}

This work was done during the visits made by the first two authors to Laboratoire de Mathématiques "Raphaël Salem" de l'Université de Rouen 
and by the third author to Dipartimento di Matematica della Seconda Università degli Studi di Napoli. Hospitality and support of all these institutions are gratefully acknowledged.

\section{REFERENCES}

[1] P. Bénilan, L. Boccardo, T. Gallouët, R. Gariepy, M. Pierre, and J.L. Vazquez. An $L^{1}$-theory of existence and uniqueness of solutions of nonlinear elliptic equations. Ann. Scuola Norm. Sup. Pisa, 22:241-273, 1995.

[2] D. Blanchard, O. Guibé, and H. Redwane. Existence and uniqueness of a solution for a class of parabolic equations with two unbounded nonlinearities. (in preparation).

[3] D. Blanchard and F. Murat. Renormalized solution for nonlinear parabolic problems with $L^{1}$ data, existence and uniqueness. Proc. Roy. Soc. Edinburgh Sect. A, 127:11371152, 1997.

[4] D. Blanchard, F. Murat, and H. Redwane. Existence and uniqueness of a renormalized solution for a fairly general class of nonlinear parabolic problems. J. Differential Equations, 177:331-374, 2001.

[5] D. Blanchard and A. Porretta. Stefan problems with nonlinear diffusion and convection. J. Differential Equations, 210(2):383-428, 2005.

[6] L. Boccardo, A. Dall'Aglio, T. Gallouët, and L. Orsina. Nonlinear parabolic equations with measure data. J. Funct. Anal., 147(1):237-258, 1997.

[7] L. Boccardo and T. Gallouët. On some nonlinear elliptic and parabolic equations involving measure data. J. Funct. Anal., 87:149-169, 1989.

[8] L. Boccardo, F. Murat, and J.P. Puel. Existence results for some quasilinear parabolic equations. Nonlinear Anal., 13(4):373-392, 1989.

[9] J. Carrillo and P. Wittbold. Uniqueness of renormalized solutions of degenerate elliptic-parabolic problems. J. Differential Equations, 156(1):93-121, 1999.

[10] G. Dal Maso, F. Murat, L. Orsina, and A. Prignet. Renormalized solutions of elliptic equations with general measure data. Ann. Scuola Norm. Sup. Pisa Cl. Sci. (4), 28(4):741-808, 1999.

[11] R. Di Nardo. Nonlinear parabolic equations with a lower order term. Commun. Pure Appl. Anal., 9(4):929-942, 2010.

[12] R. Di Nardo, F. Feo, and O. Guibé. Existence result for nonlinear parabolic equations with lower order terms. Anal. Appl. (Singap.), 2(2):161-1866, 2011.

[13] R. Di Nardo and A. Perrotta. Uniqueness results for nonlinear elliptic problems with two lower order terms. (in preparation).

[14] R.-J DiPerna and P.-L Lions. On the cauchy problem for Boltzmann equations : global existence and weak stability. Ann of Math, 130(1):321-366, 1989.

[15] R.-J DiPerna and P.-L Lions. Ordinary differential equations, sobolev spaces and transport theory. Invent. Math, 98:511-547, 1989.

[16] O. Guibé and A. Mercaldo. Uniqueness results for noncoercive nonlinear elliptic equations with two lower order terms. Commun. Pure Appl. Anal., 7(1):163-192, 2008.

[17] G. G. Lorentz. Some new functional spaces. Ann. of Math. (2), 51:37-55, 1950.

[18] F. Murat. Soluciones renormalizadas de EDP elipticas non lineales. Technical Report R93023, Laboratoire d'Analyse Numérique, Paris VI, 1993. Cours à l'Université de Séville.

[19] F. Murat. Equations elliptiques non linéaires avec second membre $L^{1}$ ou mesure. In Compte Rendus du 26ème Congrès d'Analyse Numérique, les Karellis, 1994.

[20] Richard O'Neil. Integral transforms and tensor products on Orlicz spaces and $L(p, q)$ spaces. J. Analyse Math., 21:1-276, 1968.

[21] F. Petitta. Renormalized solutions of nonlinear parabolic equations with general measure data. Ann. Mat. Pura Appl. (4), 187(4):563-604, 2008. 
UNIQUENESS OF RENORMALIZED SOLUTIONS TO NONLINEAR PARABOLIC . . 25

[22] M. M. Porzio. Existence of solutions for some "noncoercive" parabolic equations. Discrete Contin. Dynam. Systems, 5(3):553-568, 1999.

[23] A. Prignet. Existence and uniqueness of "entropy" solutions of parabolic problems with $L^{1}$ data. Nonlinear Anal., 28(12):1943-1954, 1997.

[24] H. L. Royden. Real analysis. Macmillan Publishing Company, New York, third edition, 1988.

[25] J. Serrin. Pathological solution of elliptic differential equations. Ann. Scuola Norm. Sup. Pisa Cl. Sci., 18:385-387, 1964.

Dipartimento di Matematica, Seconda Università Degli Studi di Napoli, VIALE LinCOLN, 5 - 81100 CASERTA, ITALY

E-mail address: Rosaria.Dinardo@unina.it

Dipartimento per le Tecnologie, Università degli Studi di Napoli Parthenope, Centro Direzionale Isola C4, 80100 Naples, Italy

E-mail address: Filomena.Feo@uniparthenope.it

Laboratoire de Mathématiques Raphä̈l Salem, Université De Rouen, CNRS, F-76801 Saint Etienne DU Rouvray CEDEx, France

E-mail address: Olivier.Guibe@univ-rouen.fr 